\title{
Effect of phosphorus supplementation on diet induced thermogenesis of high protein-low phosphorus meal
}

\author{
L. Abdouni ${ }^{1}$, A. Olabi ${ }^{1}$, M. Bassil ${ }^{2}$ and O. Obeid ${ }^{1}$ \\ ${ }^{1}$ Department of Nutrition and Food Science, American University of Beirut and ${ }^{2}$ Department of Natural Sciences, \\ Lebanese American University, Beirut, Lebanon
}

Diet induced thermogenesis (DIT) of proteins is known to be higher than that of carbohydrates and fats (1). Proteins contain high quantity of phosphorus that is required for energy production in the form ATP, which is a major contributor to DIT. However, it is not clear whether DIT of proteins is affected by their content of phosphorus. Thus, a study was conducted to explore the impact of phosphorus on DIT of protein using a low phosphorus containing protein, egg white.

A crossover design study (Approved by AUB IRB) was performed where nine healthy lean male subjects undertook 2 experimental sessions separated by a minimum of one week. Overnight fasted subjects were asked to consume a 554 Kcal high protein-low phosphorus (using egg white) meal (E \% from protein $50 \%$ ) with phosphorus $(500 \mathrm{mg}$ ) or placebo tablets in a random order. Energy expenditure was measured at baseline and for the next four hours in a period of 15 minutes interval with 15 minutes break, using a ventilated hood and canopy system (COSMED QUARK CPET UNIT) for indirect calorimetry measurement. Changes in energy expenditure from baseline were calculated and data were analyzed using two way analysis of variance (ANOVA).

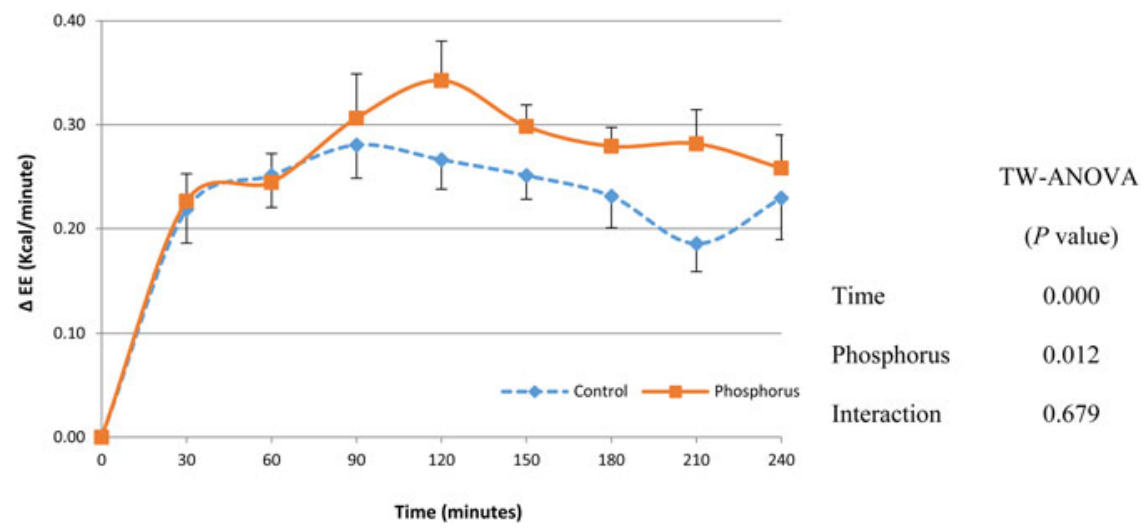

Fig. 1. Change in Postprandial Energy Expenditure

Postprandial energy expenditure increased following the ingestion of the different meals $(\mathrm{P}=0 \cdot 000)$ and did not go back to baseline level within the 4 hour experimental period. Postprandial energy expenditure of the phosphorus supplemented group was found to be higher than that of the control (Fig. 1; $P=0.012$ ) especially from time $120 \mathrm{~min}$ and onward. In conclusion, our result implies that phosphorus is involved in the high thermic effect of proteins.

The work was supported by a grant from the University Research Board at the American University of Beirut.

1. Flatt JP. The biochemistry of energy expenditure. In: Bray GA, editor. Recent advances in obesity research. London: Newman; 1978. p. 211-28. 